\title{
Tratamento do câncer de próstata: radioterapia, quimioterapia e plantas medicinais como alternativa terapêutica
}

\author{
Treatment of prostate cancer: radiotherapy, chemotherapy and medicinal plants as a therapeutic \\ alternative
}

Tratamiento del cáncer de próstata: radioterapia, quimioterapia y plantas medicinales como alternativa terapéutica

Rayane Siqueira de Sousa ${ }^{1 *}$, Ângela Magalhães Vieira1, Isabel Michely da Silva Galvão de Melo ${ }^{1}$, Thiago Felix da Silva1', Ivone Antônia de Souza¹.

\section{RESUMO}

Objetivo: Este artigo tem como objetivo elaborar uma revisão da literatura com estudos relevantes, sobre o tratamento de câncer de próstata $(\mathrm{CaP})$ envolvendo radioderapia, quimioterapia e plantas medicinais. Metodologia: Foi realizada uma revisão de literatura nas bases de dados: Scientific Eletronic Library Online (SCIELO), PubMed e nos periódicos CAPES, utilizando os descritores: câncer de próstata, plantas medicinais, radioterapia e quimioterapia, publicados entre 2010 e 2018. Resultados: Radioterapia (RT) é um método que utilizam feixes de radiações ionizantes capaz de destruir ou impedir a proliferação de células tumorais. Sendo assim, novas formas de terapias nesta área estão surgindo como: a exclusão androgênica, CaP localizado, a RT guiada por imagem, RT estereotáxica ablativa e a RT conformada tridimensional. Já a quimioprevenção é a maneira mais indicada, pois, reduz as chances de desenvolvimento de tumores a partir da utilização de medicamentos. No caso do CaP, o conjunto de drogas quimiopreventivas com maior eficiência em testes clínicos inclui as que apresentam inibidores da enzima 5-alfa redutase, que convertem a testosterona em diidrotestosterona. Em relação à fitoterapia, estima-se que $60 \%$ dos anticancerígenos comercializados, ou em investigações clínicas, são derivados de origem natural como Crocus sativus, Euphorbia zovitsii, Achillea wilhelmsii, Urtica dioica, Medicago sativae, Psoralea corylifolia. Conclusão: Dentro desse contexto, foi possível evidenciar que todas essas terapias citadas no texto são ferramentas importante para o tratamento do câncer de próstata, enfatizando que a fitoterapia representa uma esperança de tratamento com menor efeito colateral para o homem.

Palavras-chave: Câncer de próstata, Plantas medicinais, Radioterapia, Quimioterapia.

\begin{abstract}
Objective: This article aims to elaborate a review of the literature with relevant studies on the treatment of prostate cancer ( $\mathrm{CaP}$ ) involving radioderapia, chemotherapy and medicinal plants. Methods: A review of the literature was conducted in the databases: Scientific Electronic Library Online (SCIELO), PubMed and CAPES journals, using the descriptors: prostate cancer, medicinal plants, radiotherapy and chemotherapy, published between 2010 and 2018. Results: Radiotherapy (RT) is a method that uses beams of ionizing radiation capable of destroying or preventing the proliferation of tumor cells. Thus, new forms of therapies in this area are emerging, such as: androgenic exclusion, localized $\mathrm{CaP}$, image-guided RT, ablative stereotactic RT, and three-dimensional conformal RT. Since chemoprevention is the best way, therefore, it reduces the chances of developing tumors from the use of medicines. In the case of $\mathrm{CaP}$, the most chemopreventive drugs in clinical trials include those with 5-alpha reductase inhibitors that convert testosterone to dihydrotestosterone. In relation to phytotherapy, it is estimated that $60 \%$ of marketed anticancer drugs, or in clinical investigations, are derivatives of natural origin such as Crocus sativus, Euphorbia zovitsii, Achillea wilhelmsii, Urtica dioica,
\end{abstract}

${ }^{1}$ Universidade Federal de Pernambuco - UFPE, Recife-PE. *E-mail: rayanesiqueira@hotmail.com.br 
Medicago sativae, Psoralea corylifolia. Conclusion: Within this context, it was possible to show that all of these therapies cited in the text is an important tool for the treatment of prostate cancer, emphasizing that phytotherapy represents a hope for treatment with a lower side effect for men.

Keywords: Prostate cancer; Medicinal plants; Radiotherapy; Chemotherapy.

\section{RESUMEN}

Objetivo: Este artículo tiene como objetivo elaborar una revisión de la literatura con estudios relevantes, sobre el tratamiento de cáncer de próstata $(\mathrm{CaP})$ envolviendo radioderapia, quimioterapia y plantas medicinales. Metodología: se realizó una revisión de literatura en las bases de datos: Scientific Eletronic Library Online (SCIELO), PubMed y en los periódicos CAPES, utilizando los descriptores: cáncer de próstata, plantas medicinales, radioterapia y quimioterapia, publicados entre 2010 y 2018. Resultados: Radioterapia (RT) es un método que utiliza haces de radiaciones ionizantes capaces de destruir o impedir la proliferación de células tumorales. Siendo así, nuevas formas de terapias en esta área están surgiendo, como: la exclusión androgénica, CaP localizado, la RT guiada por imagen, RT estereotáxica ablativa y la RT conformada tridimensional. La quimioprevención es la manera más indicada, pues, reduce las posibilidades de desarrollo de tumores a partir del uso de medicamentos. En el caso del $\mathrm{CaP}$, el conjunto de drogas quimiopreventivas con mayor eficiencia en ensayos clínicos incluye las que presentan inhibidores de la enzima 5-alfa reductasa, que convierten la testosterona en dihidrotestosterona. En cuanto a la fitoterápia, se estima que el $60 \%$ de los anticancerígenos comercializados, o en investigaciones clínicas, son derivados de origen natural como Crocus sativus, Euphorbia zovitsii, Achillea wilhelmsii, Urtica dioica, Medicago sativae, Psoralea corylifolia. Conclusión: Dentro de ese contexto, fue posible evidenciar que todas esas terapias citadas en el texto es una herramienta importante para el tratamiento del cáncer de próstata, enfatizando que la fitoterapia representa una esperanza de tratamiento con menor efecto colateral para el hombre.

Palabras clave: Cáncer de próstata; Plantas medicinales; Radioterapia; Quimioterapia.

\section{INTRODUÇÃO}

O câncer de próstata $(\mathrm{CaP})$ representa o quarto principal câncer da população geral, com incidência de $7,9 \%$. No Brasil, é o segundo mais comum entre os homens, atrás apenas do câncer de pele, e tem estimativa de 68.220 novos casos em 2018, de acordo com a Agência Internacional de Pesquisa sobre o Câncer (IARC) (ASADI-SAMANI M, et al., 2018).

O diagnóstico é feito através de três principais exames: exame do toque digitálico da próstata, por meio do toque retal, que busca palpar nódulos enrijecidos; o exame de Dosagem Sérica do antígeno prostático específico PSA, que resulta em mensurar o tamanho da próstata e progressão da doença; e o ultrassom transretal (UTR), com a capacidade de mostrar lesões que causam pouco eco no sonograma (HEIDENREICH $A$, et al. 2014). O tratamento para o CaP envolve combinação de cirurgia, radiação e quimioterapia. No entanto, apesar desses tratamentos, a resistência à drogas, complicações medicamentosas e efeitos adversos, são bastante presentes, não sendo capaz de reduzir as taxas de mortalidade de pacientes com câncer de próstata, permanecendo abaixo do ideal (GRAHAM L, et al., 2018).

Devido aos tratamentos quimioterápicos que atualmente estão sendo empregados, especialmente em câncer de próstata metastático, existe a necessidade de novos fármacos mais eficientes e específicos, causando menos efeitos colaterais e de baixo custo. Nos últimos anos, tem crescido o interesse de produtos naturais para o uso terapêutico, algumas plantas medicinais descritas, são até hoje, relevantes na medicina moderna. Embora alguns materiais vegetais já sejam utilizados como medicamentos, continuam a ser importantes fontes de princípios ativos purificados que se tornaram fundamentais para a terapia moderna (TABASSUM N e HAMDANI M, 2014). As plantas medicinais são estruturalmente diversas e servem como fonte de estudo no desenvolvimento de novos fármacos (SCHUFFENNHAUER A e VARIN T, 2011). 
O tratamento quimioterápico e radioterápico aumenta a sobrevida livre da doença, uma vez que são tratamentos que promovem o controle ou a erradicação de micrometástases. Porém, estão associados a efeitos colaterais, muitas vezes, agressivos que podem conduzir o paciente a alterações na sua autoestima, perda funcional, além de alterações emocionais e sociais. (BONASSA EMA, 2012).

A presença de diferentes espécies vegetais e o fácil acesso são fatores que levam as pessoas a uma preferência pela medicina alternativa. O baixo custo também pode ser uma das vantagens para as populações de baixa renda. No entanto, as plantas medicinais com seus princípios ativos também são responsáveis por intoxicações e reações adversas, que podem se apresentar devido ao seu uso em doses inadequadas e/ou por períodos prolongados. A falta de informação sobre os possíveis riscos e benefícios do uso de plantas medicinais pode ser considerado um dos principais fatores que contribuem para a automedicação. (MORAES LG, et al., 2011).

Sendo assim, esse artigo tem como objetivo mostrar estudos relevantes sobre o tratamento de CaP envolvendo radioterapia, quimioterapia, assim como pesquisas sobre plantas medicinais para o tratamento alternativo.

\section{METODOLOGIA}

Foi realizada uma revisão de literatura nas bases de dados: Scientific Eletronic Library Online (SCIELO), Biblioteca Nacional de Medicina e Instituto Nacional de Saúde (PubMed) e nos periódicos CAPES, utilizando os seguintes descritores: câncer de próstata, plantas medicinais, radioterapia e quimioterapia, publicados entre os anos de 2010 e 2018. Foram incluídos nesta revisão, artigos originais e de revisão, envolvendo todas os tipos de tratamentos no câncer de próstata. Foram excluídos artigos que envolvia tratamentos relacionados a outro tipo de câncer.

\section{RESULTADOS E DISCUSÃO}

\section{RADIOTERAPIA NO TRATAMENTO DO CÂNCER DE PRÓSTATA}

A radioterapia (RT) é um método que utilizam feixes de radiações ionizantes capaz de destruir ou impedir a proliferação de células tumorais (INCA, 1993). O câncer de próstata (CaP) localizado, possui como tratamentos principais e efetivos a radioterapia definitiva e a prostatectomia radical, porém este último é raramente proposto em idosos. Vários estudos mostraram que aproximadamente um terço da metade dos pacientes submetidos à RT desenvolve uma recaída bioquímica (FICHTNER J, 2000). Porém quando compararam a RT adjuvante com a recidiva bioquímica, em três ensaios clínicos randomizados de caráter observacional com mais de 1700 pacientes, foi visto um benefício significativo para RT adjuvante na sobrevida livre da recidiva bioquímica (BOLLA M, et al., 2012)

Novas formas de terapia estão surgindo como a exclusão androgênica (terapia de privação androgênica (ADT) que quando combinada com a RT ocorre uma supressão androgênica associada a um aumento da astenia e diminuição do funcionamento social, físico e cognitivo nos dois meses seguintes a irradiação (BEKELMAN JE, et al., 2015).

No CaP localizado a RT guiada por imagem representa uma modalidade primária de tratamento, indicada para auxiliar em tratamentos que requerem extrema precisão e tem como objetivo melhorar a acurácia através de imagens obtidas na máquina antes da aplicação, tornando viável a diminuição das margens ao redor do tumor e, com isso, possibilitar novas abordagens clinicas (HAMDY FC, et al., 2016). Alguns estudos mostraram redução de mortalidade no câncer de próstata de alto risco e melhora da radiossensibilização com a combinação de terapia de privação de andrógeno com RT guiada por imagem (BOLLA M, et al., 2016).

Outra opção de tratamento neste tipo de câncer em caso de metástase é a RT estereotáxica ablativa, uma abordagem que aumenta a efetividade biológica da dose com baixas taxas de complicação. Estudos radiobiológicos demonstraram em animais de experimentação que o emprego de altas doses aumenta os danos à célula tumoral e impede a repopulação neoplásica, um dos fenômenos mais importantes relacionados 
a recidiva local (ZENG J, et al., 2014). Além de ser uma terapia alternativa, ambulatorial, não-invasiva, de curta duração (1 semana), geralmente sem sedação ou anestesia, permitindo o retorno imediato do paciente às atividades cotidianas (OST P, et al., 2016).

A RT conformada tridimensional (3DCRT) é uma das opções terapêuticas no tratamento de CaP, estudos mostraram resultados favoráveis com escalonamento de dose de irradiação, mas em altas doses estão associadas a um risco aumentado de toxicidade aguda e tardia do organismo (MOON D, et al., 2016; ZIETMAN AL, et al., 2010). Para diminuir a irradiação de órgãos em risco, permitindo que haja o aumento da dose para o volume alvo, novas tecnologias, como a radioterapia de intensidade modulada (IMRT) e orientação de imagem foram introduzidas na prática clínica (MAUND IF, et al., 2014).

Há muitas controvérsias sobre as taxas de toxicidade entre a RT adjuvante e RT de resgate, porém as maiorias dos estudos mostraram que ambas possuem o grau de toxicidade semelhante, como o estudo de Cozzarini C. et al. (2012), que comparou a toxicidade tardia em 556 pacientes tratados com RT adjuvante versus 186 pacientes do grupo RT de resgate. Os resultados mostraram toxicidades de grau 2 e 3 idênticas para ambos os grupos. Por outro lado, Sowerby RJ et al. (2014) compararam a toxicidade da RT adjuvante precoce (<6 meses; $n=162)$ e tardia ( $\geq 6$ meses; $n=490)$. As taxas de efeitos colaterais como incontinência urinária, estenose uretral e contratura do colo da bexiga em 3 anos foram semelhantes em ambos os grupos. Hegarty SE et al. (2015) analisaram pacientes recebendo RT adjuvante $(n=894)$ versus RT de resgate $(n=$ 734) versus RT sozinha $(n=4509)$. Onde observaram que a $R T$ sozinha com a $R T$ adjuvante foi associada a um risco maior de efeitos colaterais gastrointestinais e que as taxas de toxicidade geniturinário (GU) não foram significativamente maiores na RT adjuvante do que no grupo RT de resgate.

\section{QUIMIOTERAPIA NO TRATAMENTO DO CÂNCER DE PRÓSTATA}

A quimioprevenção é a maneira de reduzir as chances de desenvolvimento de tumores a partir da utilização de medicamentos (STEWARD WP e BROWN K, 2013). No caso do câncer de próstata, o conjunto de drogas quimiopreventivas com maior eficiência em testes clínicos inclui as que apresentam inibidores da enzima 5alfa redutase, que convertem a testosterona em diidrotestosterona (SILVA MHA, et al., 2018). Dentre estes fármacos estão a Finasterida e a Dutasterida, que em ensaios clínicos reduziram em $24,8 \%$ e $22,8 \%$ respectivamente, as chances de desenvolver CaP quando comparadas com o grupo placebo (FERRíS-ITORTAJADA J, et al., 2011). Estudos que envolviam suplementos como vitamina $E$, ácido fólico, selênio e vitamina $C$ também tiveram seus efeitos comparados ao placebo, porém não demonstraram redução na incidência da doença (STROPE SA e ANDRIOLE GL, 2010).

A quimioterapia é indicada quando o quadro clínico corresponde a metástases sintomáticas (MOTTET N, et al., 2011). Medicamentos como a goserelina, a triptorelina e a leuprorelina são análogas ao hormônio liberador de gonadotropina e bloqueiam a liberação de andrógenos. O docetaxel e o cabazitaxel são agentes citostáticos que impedem a formação do fuso mitótico durante a mitose. $\mathrm{O}$ acetato de abiraterona inibe a enzima 17 alfa-hidroxilase / C17,20-lyase (CYP 17) necessária para a biossíntese de andrógenos. A enzalutamida inibe receptores de andrógenos. Os principais efeitos desses quimioterápicos em pacientes com CaP incluem vômitos, diarréia, alopecia e a neutropenia a nível hematológico (BORAU PG, et al., 2017).

Pacientes que se encontram com o $\mathrm{CaP}$ em estágio avançado devem ser tratados com docetaxel, pois geralmente apresentam metástase em órgãos como fígado e pulmão, porém é mais frequente no esqueleto (MOTTET N, et al., 2011). A metástase óssea está associada a morbidez e inclui complicações como fraturas e compressão medular. Para reduzir a proliferação dos tumores através da apoptose dos osteoclastos são utilizados medicamentos do tipo bisfosfonatos (DOTTO ML e DOTTO AC, 2011). O ácido zoledrônico pertencente a esta classe, foi administrado em pacientes através de uma dose de $4 \mathrm{mg}$ a cada 3 semanas, por 15 meses. Em comparação ao grupo placebo, os resultados incluíram redução de dores e fraturas, sem diferenças significativas na taxa de sobrevida (GOMEZ-VEIGA F, et al., 2013).

REAS/EJCH | Vol. 11 (9) | e537 | DOI: https://doi.org/10.25248/reas.e537.2019 Página 4 de 7 
Em pacientes com CaP metastático resistente a castração, o tratamento é realizado com docetaxelprednisona (MOROTE J, et al., 2016). Em pacientes com CaP resistente a hormonioterapia, o tratamento de primeira linha é realizado com o regime de docetaxel-prednisona. A administração de carbazitaxel-prednisona ou acetato de abiraterona corresponde a segunda linha. Ambos os tratamentos para pacientes hormônio resistentes têm o objetivo de melhorar a qualidade de vida e aumentar a taxa de sobrevida, mas após a segunda linha não há comprovação de benefícios na sobrevida (MARTíNEZ BMA, et al., 2013).

Um estudo para avaliar a eficácia da quimioterapia sistêmica baseada em docetaxel em pacientes coreanos com câncer de próstata resistente à castração (CRPC) foi realizado com 30 idosos divididos em dois grupos. O primeiro formado por 9 pacientes com idade abaixo de 70 anos e o segundo por 21 pacientes com idade igual ou superior a 70 anos. Ambos receberam tratamento de prednisona $(5 \mathrm{mg})$ duas vezes ao dia e docetaxel $\left(75 \mathrm{mg} / \mathrm{m}^{2}\right)$ a cada três semanas. A toxicidade não diferiu entre os grupos e o bom estado funcional da quimioterapia mostrou que o tratamento com docetaxel é bem tolerado por pacientes acima de 70 anos (PARK SCH, et al., 2012). Outro estudo com 45 pacientes turcos com CRPC foi realizado para avaliar a eficácia do docetaxel em um tratamento de $75 \mathrm{mg} / \mathrm{m}^{2}$ administrado uma vez a cada três semanas e mostrouse eficiente para aliviar os sintomas da doença e prolongar a sobrevida (MANGIR N e TÜRKERI L, 2014).

O sunitinib, um fármaco utilizado no tratamento de pacientes com tumores do estroma gastrointestinal e câncer renal por apresentar ação antiangiogênica e antiproliferativa de células tumorais, foi testado em pacientes com câncer de próstata metastático refratário a hormônios e que não respondiam ao docetaxel. Neste estudo, dos 8 pacientes que passaram por administração de $50 \mathrm{mg}$ em ciclos de quatro semanas de tratamento com duas semanas fora do ciclo, para um total de oito ciclos, 5 tiveram redução do PSA. Os efeitos colaterais mais comuns incluíram astenia, diarreia, anemia e trombocitopenia. (GASENT JM, et al., 2011).

\section{PLANTAS MEDICINAIS COMO TRATAMENTO ALTERNATIVO}

A comprovação de substancia fitoquímicas com ação anticancerígena significativa iniciou com os alcalóides (vincristina e vinblastina), isolados da Vinca rosea. Estes alcalóides e seus derivados semisintéticos bloqueiam a mitose no estágio de metáfase e induz a apoptose. Outros fitoquímicos de grande importância na terapia do câncer são os derivados da camptotecina (topotecano e irinotecano), epipodofilotoxina (etoposídeo e teniposídeo) e os taxanos (docetaxel, paclitaxel e cabazitaxel) (JUÁREZ P, 2014). Estima-se que $60 \%$ dos anticancerígenos comercializados, ou em investigações clínicas, são derivados de origem natural (WANG MW, et al., 2007). Extratos obtidos de plantas e seus isolados têm demonstrado resultados promissores na atividade anticancerígena contra vários tipos de linhagens de câncer de próstata (D'ALESSANDRO AM, et al., 2013; ASADI-SAMANI M, et al., 2018; LIN C, et al., 2018; FORT RS, et al., 2018).

O Crocus sativus $L$ conhecido como açafrão, uma especiaria utilizada na culinária como corante e aromatizante, apresenta três principais metabolitos secundários com atividade antineoplásica: crocina, picrocrocina e safranal. A crocetina, substância derivada da hidrólise da crocina, tem apresentado atividade anticancerígena no tratamento, e na prevenção de uma grande variedade de câncer (KHORASANCHI Z, et al., 2018). Em estudo desenvolvido em camundongos nude machos, foram comparados os efeitos da crocina, crocetina e do extrato do açafrão. Neste estudo a crocetina, apresentou maior efeito antitumoral quando comparada com a crocina e o extrato de açafrão. Nesses três tratamentos, foram suprimidas a transição epitelial-mesenquimal (EMT) e inibida a invasão e a migração de células cancerígenas através da regulação negativa da expressão e atividade das metaloproteinases e urocinases (FESTUCCIA C, et al., 2014). Nos cultivos de células derivadas de câncer de próstata, tanto o extrato de açafrão quanto a crocina, demonstraram inibir a proliferação celular, impedindo a progressão do ciclo celular, ativando uma via apoptótica intrínseca via caspase-9 (D’ALESSANDRO AM, et al., 2013).

$\mathrm{Na}$ análise da atividade inibitória de plantas medicinais iranianas em linhagens celulares de câncer de próstata (PC-3 e DU-145), Euphorbia zovitsii, Achillea wilhelmsii, Urtica dioica e Medicago sativa apresentaram atividade anti-proliferativa para uma ou ambas as linhagens de células cancerícegenas (ASADISAMANI M, et al., 2018)

REAS/EJCH | Vol. 11 (9) | e537 | DOI: https://doi.org/10.25248/reas.e537.2019 Página 5 de 7 
Psoralea corylifolia (Fabaceae) é amplamente distribuída na Índia e Sul da Ásia e popularmente utilizada na medicina tradicional local. Entre seus principais compostos bioativos, estão o psoraleno e o isopsoraleno. $O$ psoraleno inibe o crescimento in vitro de vários tipos de linhagens celulares de câncer humano e 0 Isopsoraleno apresenta atividade estrogênica e antioxidante (MOHAMMAD PB, et al., 2015). O extrato etanólico de $P$. corylifolia demonstrou induzir apoptose e autofagia além de alterar a expressão gênica avaliada por microarranjo de cDNA em células PC-3 de câncer de próstata humano (LIN C, et al., 2018).

Em estudo da inibição de crescimento in vitro da linhagem de células LNCaP, algumas espécies de plantas utilizadas na medicina tradicional maia apresentaram moléculas com atividade citotóxica/antiproliferativa, entre elas: Cnidoscolus chayamansa (Euphorbiaceae), Capsicum chinense (Solanaceae), Coccoloba uvifera (Polygonaceae), Leucaena leucocephala (Fabaceae), Malmea depressa (Annonaceae) e Terminalia catappa (Combretaceae). Dois novos ácidos graxos simples com atividade antineoplásica in vitro foram identificados a partir deste estudo: éster etílico do ácido 8-metil 6-nonenoico de $C$. chinense e éster metílico do ácido 8metil-6-nonanóico de $C$. chayamansa. $O$ éster etílico do ácido 8-metil 6-nonenoico demonstrou seletividade por células neoplásicas (FORT RS, et al., 2018).

Solanum nigrum L. é uma planta daninha de solos ricos em nitrogênio. Muitos de seus componentes bioativos foram investigados devido às suas amplas atividades biológicas (BUTT G, et al., 2018). A solanina, um glicoalcalóide isolado de S. nigrum L., demonstrou atividade anticancerígena substancial contra o câncer de próstata, induzindo apoptose em células DU145 e regulação negativa dos níveis de mRNA e proteína da ciclina D1, ciclina E1, ciclina dependente quinase 2 (CDK2), CDK4 e CDK6, em tecidos tumorais de camundongos xenoenxertados com células DU145, houve redução considerável do crescimento do tumor (PAN B, et al., 2016).

Euphorbia tirucalli, tem como principal constituinte de sua seiva o euphol, um álcool triterpênico tetracíclico, que apresentou propriedades anti-inflamatórias, analgésicas e anticancerígenas (DUTRA RC, et al., 2015). O euphol diminuiu a viabilidade celular em estudos in vitro de células tumorais humanas, demonstrando ser um agente antineoplásico promissor principalmente no câncer de esôfago e pâncreas seguido por células de câncer de próstata. Este estudo também revelou a inibição/redução de alguns eventos (proliferação e migração) como parte do mecanismo de ação deste composto nas células cancerosas pancreáticas, bem como interações sinérgicas com drogas quimioterápicas usadas na prática clínica (SILVA VAO, et al., 2017).

\section{CONSIDERAÇÕES FINAIS}

Dentro desse contexto, foi possível evidenciar que todas as terapias citadas no texto são consideradas ferramentas importantes para o tratamento do câncer de próstata. Porém a fitoterapia demonstrou ser uma das terapias com resultado bastante promissor no estudo de atividade in vitro e em modelo animal de câncer de próstata, tendo em vista os efeitos colaterais presente nas quimioterapias, radioterapias e tratamentos cirúrgicos. Sendo assim, a fitoterapia representa uma esperança no tratamento para o câncer de próstata com menor efeito colateral para o homem.

\section{REFERÊNCIAS}

1. ASADI-SAMANI M, et al. A screening of growth inhibitory activity of Iranian medicinal plants on prostate cancer cell lines. BioMedicine, 2018; 8(2): 16-21.

2. BOLLA M, et al. Short Androgen Suppression and Radiation Dose Escalation for Intermediate-and-High-Risk Localized Prostate Cancer Results of EORTC Trial 22991. J. Clin. Oncol, 2016; 34:1748-1756.

3. BOLLA M, et al. Postoperative radiotherapy after radical prostatectomy for high-risk prostate cancer: long-term results of a randomised controlled trial (EORTC trial 22911). Lancet, 2012; 380(9858): 2018-2027.

4. BUTT G, et al. Emerging themes of regulation of oncogenic proteins by Solanum nigrum and its bioactive molecules in different cancers. J Cell Biochem, 2018: 1-5.

5. D'ALESSANDRO AM, et al. Crocus sativus stigma extract and its major constituent crocin possess. 
6. DUTRA RC, et al. The antinociceptive effects of the tetracyclic triterpeneeuphol in inflammatory and neuropathic pain models: The potential role of PKC epsilon. Neuroscience, 2015; 303: 126-137.

7. FESTUCCIA C, et al. Antitumor effects of saffron derived carotenoids in prostate câncer cell models. Biomed.Res.Int, 2014; 2014: 135048.

8. GRAHAM L, et al. A phase II study of the dual mTOR inhibitor MLN0128 in patients with metastatic castration resistant prostate cancer. Invest New Drugs, 2018; 36(3): 458-467.

9. GASENT JM, et al. Experiencia com sunitiniben cáncer de próstata metastásico hormono resistentes in respuesta a docetaxel. Actas Urológicas Españolas, 2011; 35(1): 57-60.

10. HAMDY FC, et al. 10-Year Outcomes after Monitoring, Surgery, or Radiotherapy for Localized Prostate Cancer. N. Engl. J. Med, 2016; 375: 1415-1424.

11. HEIDENREICH A, et al. EAU guidelines on prostate cancer. Part 1: screening, diagnosis, and local treatment with curative intent-update. Eur Urol, 2014; 65(2): 467124 - 467137.

12. LIN C, et al. The ethanol extraction of prepared Psoralea corylifolia induces apoptosis and autophagy and alteres genes expression assayed by cDNA microarray in human prostate cancer PC-3 cells. Environmental Toxicology, 2018; 33: 770-788.

13. MOON D, et al. What is the best way to radiate the prostate in 2016? Urol Oncol, 2016; 35(2): 59-68.

14. MOROTE J, et al. Cáncer de próstata. Medicina Clínica, 2016; 146(3): 121-127.

15. OST $P$, et al. Pattern of Progression after Stereotactic Body Radiotherapy for Oligometastatic Prostate Cancer Nodal Recurrences. Clin Oncol, 2016; 28(9): 115-120.

16. PAN B, et al. Inhibition of prostate cancer growth by solanine requires the suppression of cell cycle proteins and the activation of ROS/P38 signaling pathway. Cancer Med, 2016; 5(11):3214-3222.

17. SILVA VAO, et al. In vitro screening os cytotoxic activity of euphol from Euphorbya tirucally on a large panel of human cancer-derived cell lines. Experimental and Therapeutic Medicine, 2018; 16(2): 557-556.

18. SOWERBY RJ, et al. Long-term complications in men who have early or late radiotherapy after radical prostatectomy. Can Urol Assoc J, 2014; 8(7-8): 253-258.

19. TABASSUM N, HAMDANI M. Plants used to treat skin diseases. Pharmacognosy. Reviews, 2014; 8(15): 52-60.

20. ZENG J, et al. Combination of stereotactic ablative body radiation with targeted therapies. Lancet Oncol, 2014; 15(10): 426-434. 